ATLANTA

Paper ID \#7726

\title{
Analytical Studies on S-N Curves for Some Steels
}

Prof. Somnath Chattopadhyay, Georgia Southern University 


\title{
Analytical Studies on S-N Curves for Some Steels
}

\begin{abstract}
Experimental data are often employed to obtain stress amplitude versus number of cycles to failure characteristics of materials ( S-N Curve). This activity as a laboratory component of a junior level materials science course explores a novel way to understand the fatigue failure process. The students study fatigue failure in terms of crack nucleation where a small crack grows from a zero length to a threshold crack size that depends on the applied stress level and the threshold fracture toughness (a concept that the students learn in the course). A characteristic size or a distance parameter is used in the formulation which is dependent on the endurance or the fatigue limit of the material as well as the threshold fracture toughness. The student start with a given stress range and calculate the crack nucleation life (in cycles) to find the number of cycles to failure. The input material properties needed for the exercise are the endurance limit and the threshold fracture toughness.
\end{abstract}

\section{INTRODUCTION}

As a part of the material science as well as the mechanical design areas within the mechanical engineering program, students are made aware that of the fact that for machine components such as rotating shafts, gears, crankshafts containing no preexisting cracks, the majority of life is spent in initiating or starting fatigue cracks. The fatigue process in this case is therefore described as initiation controlled.

A rotating beam fatigue test is traditionally performed as a laboratory experiment in the junior level materials course. The rotating beam fatigue test provides the experimental determination of the S-N curve. The S-N curve graphically shows the stress amplitude $(\mathrm{S})$ as a function of the number of cycles to failure $(\mathrm{N})$. This test also helps the students to understand the concept of endurance limit (or fatigue limit), as the stress level below which no fatigue failure takes place. This concept finds use in the course dealing with the design of machine components.

In the same materials science course, the students are exposed to the concept of stress intensity factor that measures the severity of an existing crack in a material in terms of its size, the applied stress and the overall geometry. Along with it they also learn the concept of fracture toughness that determines the ability of a material to resist fracture in the presence of a crack. Although the crack propagation tests are not generally performed at the junior level, the students nonetheless are familiarized with the model of crack growth per loading cycle as a function of stress intensity factor range (the so-called Paris Law), and use this to analytically estimate the number of load cycles required to grow an existing crack from an initial size to a final size. The students are made aware of the fact that Paris Law is applicable for stress intensity factor range higher than the threshold value $\Delta \mathrm{K}_{\text {th }}$, a material parameter that is available in literature $[1,2]$ 
As a complementary exercise to the rotating beam fatigue test, an analytical activity has been introduced as a lab project to obtain the S-N curves for typical steels. In this activity the students construct S-N curve analytically using the crack propagation approach. Here they make use of the phenomenon of nucleation of a small crack from the free surface as the basis for fatigue crack initiation.

The threshold crack size is dependent on the applied stress level, and a material parameter, namely the threshold fracture toughness. The formulation used in Reference [3] is used where an effective crack length is introduced to predict the propagation behavior of cracks. The effective crack length is equal to the crack length plus an amount " $1_{0}$ ", a critical distance. This value " $1_{0}$ " was stated in Reference [3] as a characteristic of the material and the material condition accounting for the non-continuum behavior of very small cracks. The stress intensity range required to propagate a crack must remain finite rather than become infinitely large when the length of the short crack is extremely small. As such, the critical distance is an empirical parameter to be regarded as a material constant. The nucleation period is characterized by the growth of micro crack from a zero length to the size corresponding to the threshold stress intensity factor range.

The characteristic size is dependent on the threshold fracture toughness as well as the endurance limit or the fatigue limit of the material. For the nucleation of micro cracks the approach in Reference [4] is used that uses experimental data for medium carbon steels and assumes that the micro crack initiation occurs immediately in fatigue. Reference [4] obtains a best-fit equation to the micro structural short crack growth data for medium carbon steels in the form of a fatigue crack growth parameter $d a / d N$ proportional to the stress range raised an exponent times the difference of the grain diameter and the crack length. In this work, the grain diameter is interpreted as the distance parameter " $l_{0}$ " from Reference [3].

\section{PROBLEM STATEMENT}

In this work, fatigue crack initiation has been studied using the concept of distance parameter. The endurance limit is characterized by the maximum value of the local stress range, which generally occurs on the surface.

It is not the entire stress field that is important, but only the stress field close to the crack tip. Traditionally, fatigue crack initiation has been treated by empirically determining the nominal stress amplitude that can be applied to a smooth specimen for an infinite number of cycles. This is the endurance stress range, $\Delta \sigma_{e}$ (twice the endurance limit, $S_{e}$ ). The application of fracture mechanics to fatigue has resulted in a definition of the threshold stress intensity factor range, $\Delta K_{t h}$, below which no detectable crack propagation occurs for cracked members. Measurement of $\Delta K_{\text {th }}$ establishes a range of crack sizes and stress amplitudes for which no further crack extension takes place. 


\section{METHODOLOGY}

The distance parameter used in El Haddad's [1] work is denoted as $l_{0}$ and is given by:

$$
l_{0}=\frac{1}{\pi}\left(\frac{\Delta K_{t h}^{l}}{\Delta \sigma_{e}}\right)^{2}
$$

And

$$
\Delta S=\Delta \sigma_{e} \sqrt{\frac{1}{1+\frac{a}{l_{0}}}}
$$

The number of cycles to initiate a crack is determined by the small crack initiation stage followed by its growth to a long crack (from zero to initiation size, $a_{t h}$ ). The initiation size (denoted by $a_{t h}$ ) for a particular stress range is determined first, and is followed by the determination of the number of cycles $\left(N_{i}\right)$ required to grow the crack from a zero length to this size. This size is determined as:

$$
a_{t h}=\frac{1}{\pi}\left(\frac{\Delta K_{t h}}{\Delta S}\right)^{2}
$$

For initiation, the equation provided by Miller [2] is employed. This is valid for medium carbon steels and will be assumed to apply for the material considered in this analysis.

This equation takes the form:

$$
\frac{d a}{d N}=1.475 \times 10^{-41}(\Delta S)^{11.49}\left(l_{0}-a\right)
$$

The above equation accounts for the early decrease in the surface crack propagation rate as a function of the applied stress range $\Delta S$ and the crack length $a$.

Integrating equation (4) from the crack lengths going from zero to $a_{t h}$, we obtain the number of cycles to initiate a crack, $N_{i}$, as:

$$
N_{i}=\frac{1}{1.475 \times 10^{-41}(\Delta S)^{11.49}} \ln \left(\frac{l_{0}}{l_{0}-a_{t h}}\right)
$$




\section{FLOWCHART FOR THE ANALYTICAL APPROACH}

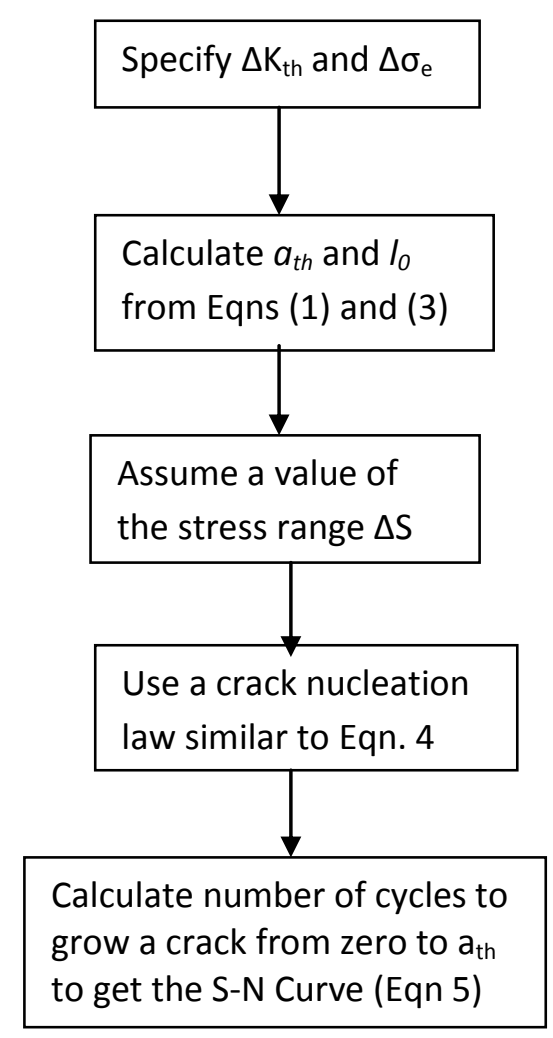

TABLE 1 Analysis Flow Chart

\section{RESULTS AND ANALYSIS}

For this assessment, the material A533B was selected for which the long crack threshold $\left(\Delta K t h^{l}\right)$ is $8 \mathrm{MPa} \sqrt{ } \mathrm{m}$. The material has an assumed endurance stress range $\left(\Delta \sigma_{e}\right)$ of $1000 \mathrm{MPa}$ (endurance limit of $500 \mathrm{MPa}$ ), the value the parameter " $l_{0}$ " from equation (1) is calculated to be $140 \mu \mathrm{m}$ or $0.02 \mathrm{~mm}$. With these values the limiting stress range for fatigue failure (Eqn 5) as a function of crack size (a) is obtained using equation (1).

For a number of stress ranges the number of cycles $N_{i}$, for initiating the crack from zero to $a_{t h}$, is calculated and the S-N curve constructed as shown in Figure 1. 


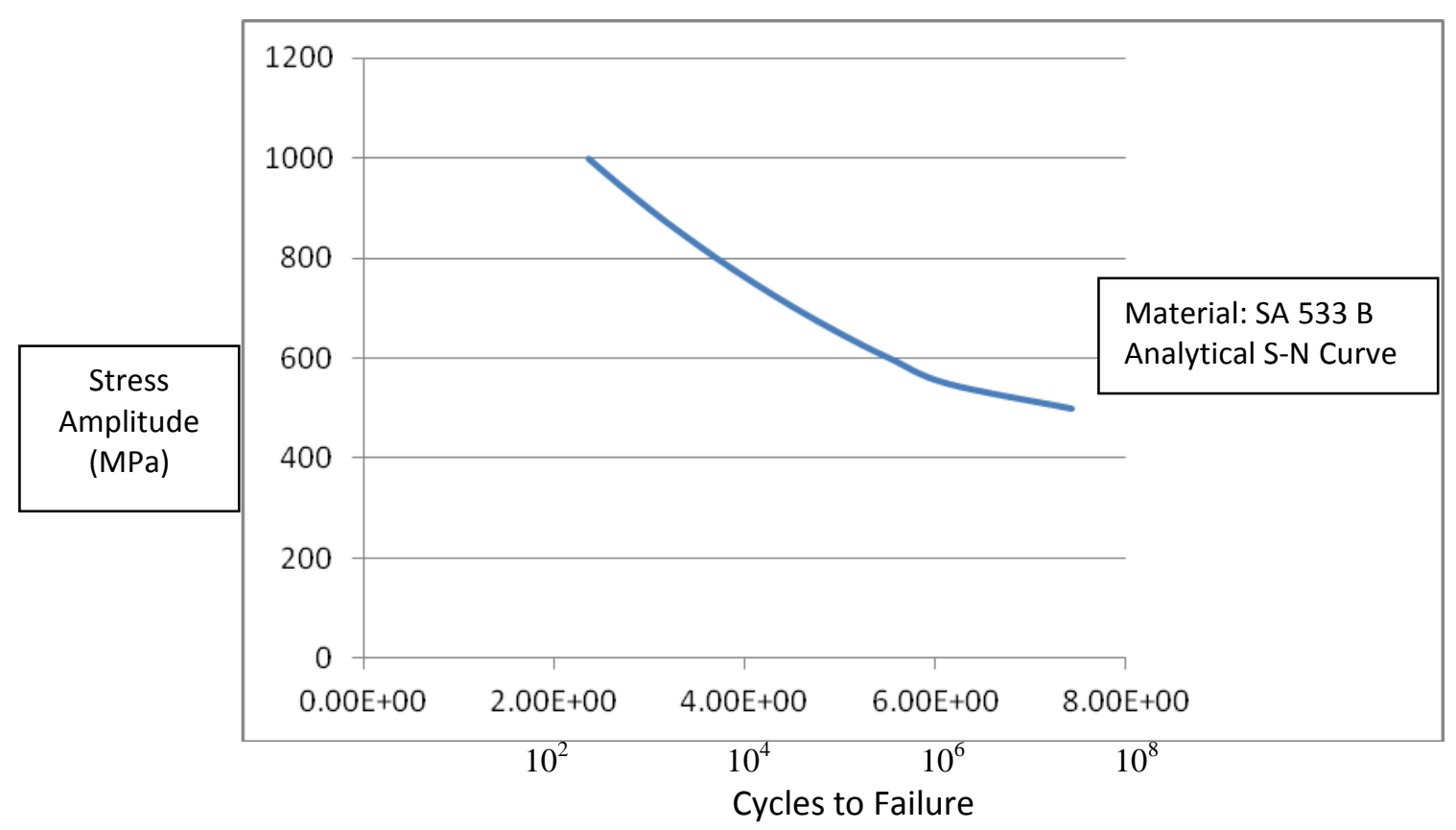

Figure 1: S-N Curve

\section{ASSESSMENT OF STUDENT LEARNING}

The students were asked to pick steels whose endurance limits (typically half the ultimate strength) and the threshold stress intensity ranges are known. For example, for a different steel (other than $533 \mathrm{~B}$ ) such as A 517 the endurance stress range $\Delta \sigma_{\mathrm{e}}=828 \mathrm{MPa}$ and the threshold stress intensity range is $\Delta \mathrm{K}_{\mathrm{th}}=8 \mathrm{MPa} \sqrt{\mathrm{m}}_{\mathrm{m}}$. [1] Adopting a similar procedure (see Table 1) the $\mathrm{S}$ $\mathrm{N}$ curve can be obtained and may be compared with the corresponding $\mathrm{S}-\mathrm{N}$ curve in Reference [1] for the same material as shown in Figure 2.

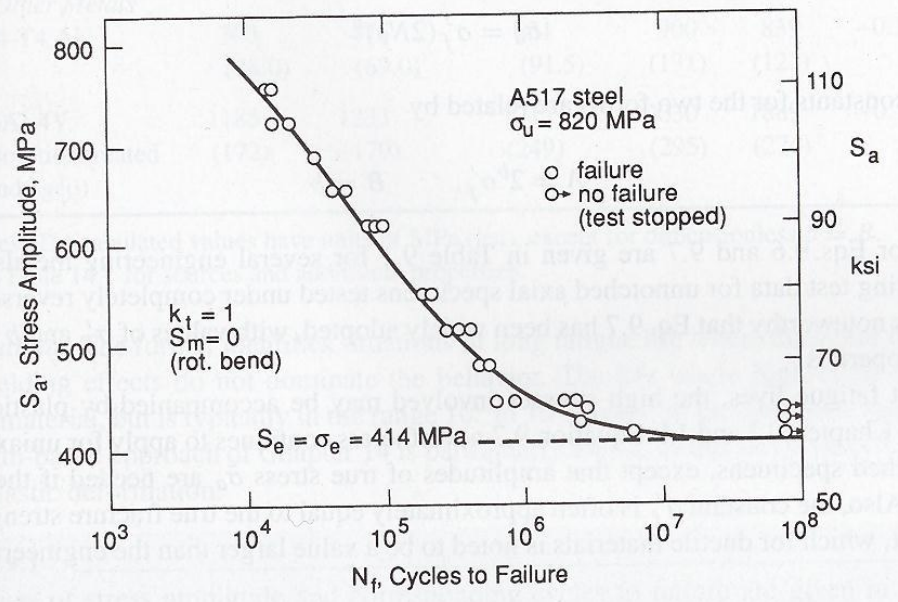

Figure 2 S-N Curve for A 517 Steel (Ref. [1])

The students are assessed for the following concepts: 
(a) What do you mean by the endurance limit?

(b) What do you mean by the stress intensity factor?

(c) What do you mean by the threshold stress intensity factor range?

(d) Do you see a connection between the endurance limit and the threshold stress intensity factor range? If so what is it?

(e) How the aspect of fatigue crack initiation treated in this assessment?

\section{CONCLUSION}

The students are made aware of the fact that most of the failures in the industrial world can be attributed to fatigue and is based on the response of the structural materials to alternating loads that are applied during service. The S-N curve, a graphical representation of the cyclic loading, is a plot of stress amplitude versus the number of cycles the material goes through before it fails. The greater the number of cycles in the loading history, the smaller the stress the material can withstand without failure.

A rational process employing the nucleation of a crack on an assumed smooth surface (from a zero to the threshold value of crack length) and its subsequent growth to a threshold value is assumed to govern the phenomenon of fatigue crack initiation.

By making use of an assumed crack initiation law (Equation 4), the S-N curve for a structural material has been obtained. Although most of the discussion in this work is directed to a typical pressure vessel steel material A 533-B, the S-N curves for other steels can be obtained using a very similar approach.

\section{BIBLIOGRAPHY}

[1] Dowling, N. E.(2007), Mechanical Behavior of Materials, Third Edition, Pearson Prentice Hal

[2] Suresh, S. (2004), Fatigue of Materials, Second Edition, Cambridge University Press.

[3] El Haddad, M. H., Dowling, N.E., Topper, T. H., and Smith. K. N. (1980), “J Integral Applications for Sort Fatigue Crack at Notches," International Journal of Fracture, 16, 15.

[4] Miller, K. J. (1985), "Initiation and Growth of Short Fatigue Cracks," Fundamentals of Deformation and Fracture, (B.A. Bilby, K.J. Miller and J. R. Willis, eds), Cambridge University Press, 477-500. 